STRUCTURAL BIOLOGY

ISSN 2059-7983

\section{ALEPH: a network-oriented approach for the generation of fragment-based libraries and for structure interpretation}

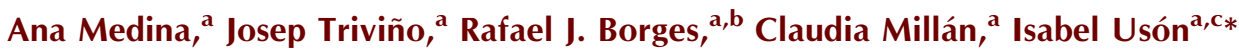 \\ and Massimo D. Sammito ${ }^{d}$ *
}

Received 29 August 2019

Accepted 5 February 2020

Keywords: X-ray phasing; bioinformatics; folding; characteristic vector; fold clustering; $A L E P H$; fragment-based libraries.

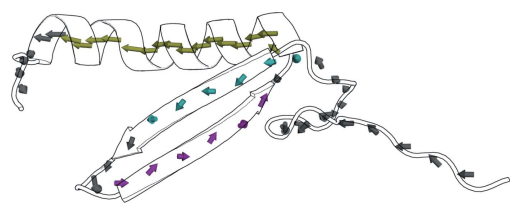

\section{Introduction}

Secondary-structure properties are usually derived from the hydrogen-bond pattern. They were predicted even before the structures of full proteins had been determined (Pauling et al., 1951; Pauling \& Corey, 1951). Analysing this network implies assessment of the environment of the amino acid in a peptide, made up of nonconsecutive residues, which may encompass symmetry equivalents that are not explicitly contained in the PDB set of coordinates. The formation of these hydrogen bonds and the planarity of the peptide bond restrict the protein backbone to adopting torsion-angle values in characteristic ranges, corresponding to the most populated areas of 
the Ramachandran plot (Ramachandran et al., 1963). Conversely, the analysis of the relevant torsion angles may suffice to characterize the secondary structure. Definition of Secondary Structure of Protein (DSSP) is the standard algorithm employed for the prediction of hydrogen positions and bonds, from which the secondary-structure environment for each residue can be derived (Kabsch \& Sander, 1983; Touw et al., 2015). Distortions in the polypeptide chain are sometimes encountered, and especially when the resolution falls below 3$3.5 \AA$ (Headd et al., 2012; Karmali et al., 2009) some structures may fail to meet DSSP regularity. DipSpace (Pereira \& Lamzin, 2017) embeds geometrical information about the backbone atoms around each $\mathrm{C}^{\alpha}$ atom in its dipeptide-unit environment, which is described as a matrix of the interatomic distances. Also, CaBLAM (Richardson et al., 2018) defines a novel parameter space of $\mathrm{C}^{\alpha}-\mathrm{C}^{\alpha}$ and $\mathrm{CO}-\mathrm{CO}$ virtual dihedrals, where the $\mathrm{CO}$ dimension diagnoses large distortions of peptide orientation at low resolution and the two $\mathrm{C}^{\alpha}$ dimensions identify the probable secondary structure obscured by these problems. CaBLAM is designed for structure validation to detect errors in the model, whereby poor geometry introduces ambiguity.

For our purposes, further abstraction can be achieved by focusing on the carbonyl bond as a lever in the necessary torsions to form hydrogen bonds. This gives rise to a characteristic atomic distribution within archetypal secondarystructure elements (Sammito et al., 2013). We denominate the vectors defined from the centroids of all $\alpha$-carbons to the centroids of all carbonyl $\mathrm{O}$ atoms in a polypeptide stretch as 'characteristic vectors' (CVs). Beyond the description of secondary structure, such vectors can be used to characterize the fold through their relative angles and distances. Also, locating them in a spatial context makes geometrical comparisons possible. The advantage of CVs is that the same reduction in dimensionality can be applied within different scopes: for example, the environment of single amino acids, when CVs are calculated over overlapping tripeptides, or to secondary-structure units in a fold, when CVs are defined over such longer stretches. This formalism is particularly useful for the geometric description of the small fragments used for phasing in the ARCIMBOLDO programs (Millán et al., 2015). Since the first implementation of the method (Rodríguez et al., 2009), combining molecular-replacement (MR) searches of small secondary-structure fragments with Phaser (McCoy et al., 2007) and density modification and autotracing with SHELXE (Sheldrick, 2010), ARCIMBOLDO has been extended to integrate other sources of information (Rodríguez et al., 2012) and diversified to use libraries of fragments (Sammito et al., 2013). Several bioinformatics tools are available to extract folds or models similar to a template structure using sequence or structural alignments. The Dali server (Holm, 2019) is a web service from which the user can obtain a sorted hit list corresponding to a specific input fold, MASTER (Zhou \& Grigoryan, 2015) defines a new r.m.s.d.-based metric to explore and extract fragments from a precomputed database and PDBeFold (Krissinel \& Henrick, 2005) is based on multiple structure alignments across families of structures.
Our approach, $A L E P H$, is designed for customizable use with small fragments. We combine the definition of new geometrical descriptors, such as CVs, with network algorithms to address fundamentally different questions. The user can control the desired strictness to accurately extract very specialized secondary-structure elements as well as general ubiquitous folds. In many applications, such a level of flexibility is fundamental to draw conclusions for different structural questions. Fragment-based MR, for example, requires a finer sampling of fold variations. In fact, characteristic vectors can be defined over shorter or longer stretches to capture fine or coarse features.

Suitable fragments from distant homologs can also be identified (Sammito et al., 2014) or improved (Millán et al., 2018) against the experimental data. CVs are used in all operations involved in identifying, extracting, comparing and annotating fragments to refine subsequent degrees of freedom (McCoy et al., 2018). CVs are also used in the verification step introduced to establish the correctness of coiled-coil solutions at low resolution (Caballero et al., 2018). Finally, we use them in the analysis of solved cases for development purposes. As phasing methods using small fragments are becoming very popular in successful pipelines such as AMPLE (Bibby et al., 2012), Fragon (Jenkins, 2018) and FRAP (Shrestha \& Zhang, 2015), and other ab initio approaches to phasing such as I-TASSER (Roy et al., 2010) and MR-Rosetta (DiMaio et al., 2011), CVs might find use in this context, where accurate structural characterization independent of the sequence is needed.

Here, we present the CV-based program $A L E P H$, which was developed as a bioinformatics tool to handle fragments and prepare libraries representing variations of a given fold for MR. Extraction of such libraries is performed without relying on sequences or alignments to allow searches across different families.

\section{Materials and methods}

\subsection{Software versions}

$A L E P H$ is written in Python 3, requiring 3.7+. The code is developed to maintain retro-compatibility with Python 2.7, although the use of a Python 3 interpreter is strongly advised whenever possible. Tutorials and documentation are available from our website (http://chango.ibmb.csic.es/ALEPH). The graphical user interface is written in Python 3 with Pyside2 and QT5. Python libraries and environment variables are managed through Conda (https://anaconda.org).

$A L E P H$ requires the libraries listed in Table 1. ALEPH is distributed through PyPI (https://pypi.org/project/pip/). From a Python 3 (https://www.python.org/) environment, installation only requires execution of the command pip install aleph.

The command alephui launches the graphical interface. The core program is also available from the command line through the command aleph. Fragment-based MR tests have always been performed through the ARCIMBOLDO 
Table 1

Summary of the Python libraries required by $A L E P H$.

\begin{tabular}{|c|c|c|}
\hline Library & Category & Reference \\
\hline NumPy 1.16 .2 & Vectorized operations on matrices and vectors & Van der Walt et al. (2011) \\
\hline Scikit-learn 0.20 .3 & Clustering and data mining & Pedregosa et al. (2012) \\
\hline BioPython 1.73 & Data handling of PDB files & Cock et al. (2009) \\
\hline CSB 1.2 .5 & Maximum-likelihood-based superposition & Kalev et al. (2012) \\
\hline Pyplot 3.0.3 & Visualization of graphs and networks & Hunter (2007) \\
\hline Python-igraph 0.7 .1 & Generation and management of networks in memory & Csardi \& Nepusz (2006) \\
\hline \multicolumn{3}{|c|}{$A L E P H$ uses the following community clustering algorithms } \\
\hline Fastgreedy & & Clauset et al. (2004) \\
\hline Infomap & & Rosvall \& Bergstrom (2008) \\
\hline Eigenvectors & & Newman $(2006 a)$ \\
\hline Label propagation & & Raghavan et al. (2007) \\
\hline Community multilevel & & Blondel et al. (2008) \\
\hline Edge betweenness & & Newman \& Girvan (2004) \\
\hline Spinglass & & Reichardt \& Bornholdt (2006) \\
\hline Walktrap & & Pons \& Latapy (2005) \\
\hline
\end{tabular}

framework (Millán et al., 2015), which relies on the Phaser intensity-based maximum-likelihood function rendering the log-likelihood gain score (Read \& McCoy, 2016) in version 2.7 and upwards, and on the correlation coefficient between observed and calculated normalized intensities (Fujinaga \& Read, 1987) as calculated in SHELXE (Sheldrick, 2002) version 2019. Phaser 2.8 was used through its CCP4 7.0 (Winn et al., 2011) or Phenix 1.17 (Liebschner et al., 2019) distributions. Structure-amplitude-weighted mean phase errors (wMPEs; Lunin \& Woolfson, 1993) were calculated with SHELXE against the models available from the PDB to assess performance. The model and maps were examined with Coot 0.8.9.1 (Emsley et al., 2010). The figures were prepared with PyMOL 2.2.0 (Schrödinger). GEPHI 0.9.2 (Bastian et al., 2009) and the free version of $y E d$ (https://www.yworks.com/) were used to read xmlgraph files and produce network pictures for this manuscript.

\subsection{Computing setup}

Library-generation tests were run on a local HTCondor version 8.4.5 (Tannenbaum et al., 2001) grid made up of 160 nodes totalling 225 Gflops. Some libraries were generated on a single workstation with two Intel Xeon E5-2680 processors totalling 24 physical cores and 128 GB RAM running Ubuntu Linux. Typical running times for library generation from the whole PDB ranged from 6 to $12 \mathrm{~h}$ on a single workstation of 24 cores. Times vary substantially depending on the nature of the fold and on the dedicated hardware. Smaller folds tend to be more general and require more computation to process the vast number of occurrences that are found. The database used for extraction may be filtered or limited to accelerate the process. Parameterization is also key: more lax, lower thresholds in the geometrical similarity to the template will increase the number of fragments to process and hence the time. Often the library produced is over-sampled for phasing purposes and needs to be clustered to eliminate redundancy. This process of reducing millions of models to tens of thousands can take one to three days.

\section{ALEPH as a composite bioinformatics tool}

Recent developments in MR have formally bound the solvability of the phase problem to an estimated LLG (eLLG; McCoy et al., 2017), allowing the minimum fractional scattering that is needed at a given accuracy to be established $a$ priori (Oeffner et al., 2013). The eLLG score is used in the fragment-based MR approach ARCIMBOLDO to guide the difficult trade-off between fragment generality and solution discrimination (Oeffner et al., 2018). While minimal fragments, such as simple secondary-structure elements, are ubiquitous across structures, their correct location usually renders a low signal. Small local folds, defined as composite sets of discontinuous secondary-structure elements (for example, three antiparallel $\beta$-strands facing two parallel helices), are still ubiquitous across different families of structures but, unlike $\alpha$-helices, cannot be represented accurately enough through a single model that will match the corresponding geometry in most unknown target structures. In this context, we developed $A L E P H$ as a bioinformatics tool to prepare libraries representing variations of a given fold for MR. The extraction of such libraries is performed without relying on sequences and alignments to allow searches across different families.

$A L E P H$ provides a convenient graphical user interface to perform four different tasks: flexible secondary-structure and tertiary-structure annotation, mapping any protein structure into a network, decomposing a structure into smaller local folds, and generating customized libraries of local folds and superposing small fragments onto complete protein structures. Fig. 1 displays the main menu of the graphical interface used to access these tasks.

$A L E P H$ performs four clearly differentiated tasks. The annotation mode writes the annotated secondary-structure elements in a PDB file and plots of the graphs describing the geometrical properties of the CVs (as PNG files). The decomposition outputs a PDB file with a different chain identifier for each group. These coordinate files are ready to be used by ARCIMBOLDO or Phaser to perform gyre and gimble refinement of the model (McCoy et al., 2018). The 
library generation places all extracted folds superposed on the reference template in a new directory library. If clustering is performed, an additional clusters directory is output. The superposition mode writes the PDB file of the superposed target structure. Any ALEPH run collects all of the output needed for the graphical interface to show the results in running time. The format of this file is standard JSON so it can be inspected programmatically.

\subsection{Secondary- and tertiary-structure annotation}

All algorithms in $A L E P H$ rely on the geometrical representation of the main chain of a protein using a discrete distribution of CVs. Originally introduced in the first implementation of ARCIMBOLDO_BORGES (Sammito et al., 2013), $A L E P H$ recasts their use in networks. For any peptide of at least three residues, a $\mathrm{CV}$ is uniquely identified as the vector connecting the geometric centroid of all $\mathrm{C}^{\alpha}$ atoms to the centroid of the $\mathrm{O}$ involved. The main chain is annotated for all possible tripeptides with an overlapping window of one residue. These vectors provide a smoothed description of the protein backbone, revealing local main-chain distortions from an idealized secondary structure. Each residue is then associated with at least one $\mathrm{CV}$ and is annotated with the probability of its being part of a helix, a strand or a coil region. The relationships between CVs are described with a complete graph network.

While a single secondary-structure annotation may suffice for general purposes, we have encountered the need to control the strictness with which we want to query secondary structure and the need to formulate the alternative questions: 'From which secondary structure is a distorted fragment derived?' or 'How close is one local fold to another?' In the context of fragment-based MR, such questions underlie decisions on how to extract or decompose models and which degrees of freedom to confer. Thus, when defining the local geometry and conformation of a small local fold it might be desirable to explore different annotations. Our aim in $A L E P H$ is to provide real-time, graphical control over different levels of annotation, smoothly relaxing restraints to ideal fragments and allowing the interactive tailoring of parameterization to a particular purpose.

Open Save Menu Settings

\section{Secondary structure annotation}

Identify archetypal secondary structures through geometrical descriptors

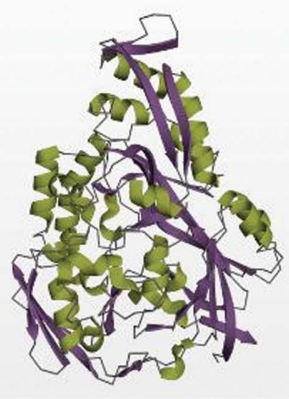

\section{Library generation}

Define, extract, cluster and superpose libraries of local folds

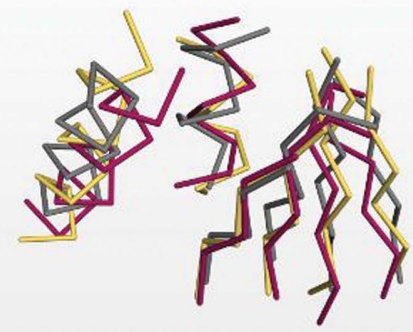

\section{Decomposition}

Cluster secondary structure elements into compact rigid groups

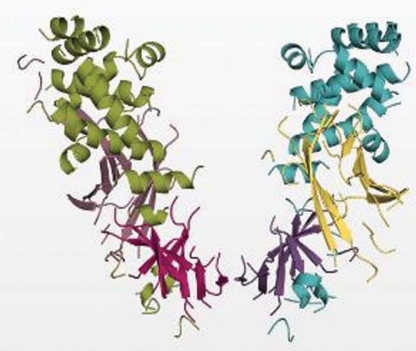

\section{Superposition}

Superpose a library of fragments into a model

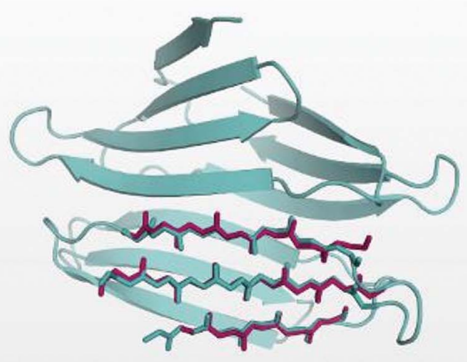

Current directory: /localdata/pep/ALEPH/aleph/gui

Figure 1

Main menu in the graphical user interface accessing the four functions in $A L E P H$ : secondary-structure annotation with graphs, fold decomposition through community clustering, template-based library generation and superposition of small fragments. 
3.1.1. Implementation. The annotation algorithm starts from the computation of $\mathrm{CVs}$ and proceeds to the iterative interpretation of their secondary and tertiary structures. The algorithm ends with the unequivocal association of a secondarystructure type, or coil, with each residue in the main chain. The general workflow is shown in Fig. 2. ALEPH maps main-chain structure into a mathematical model using as generic geometrical descriptors overlapping CVs generated with a window size of one residue. Not only secondary structure but also coils or conserved loops can be queried and compared (Pröpper et al., 2014). The geometrical relationships among these vectors are stored in a sparse matrix that can be compared against similarly annotated matrices to extract local folds.

A structure is input through a standard PDB format file. The Biopython library is used to validate the format and parse the sequence, coordinates, occupancies and $B$ factors from the PDB file. No secondary-structure annotation is imported. Filtering on occupancy reduces disordered residues to a single conformation and only residues containing all main-chain atoms are stored. Water molecules are also discarded. Connectivity between residues relies on a distance test between $\mathrm{N}$ and $\mathrm{C}$ atoms rather than on residue identifiers. For a stretch of polypeptide chain, a CV is defined with its origin at the centroid of all of its $\mathrm{C}^{\alpha}$ atoms and its end at the centroid of all carbonyl $\mathrm{O}$ atoms. A minimum number of three residues is needed to describe secondary-structure features. To determine whether this minimum number was also the optimal number, a statistical analysis was conducted against a pool of 18646 structures determined by X-ray crystallography to resolutions of $2.1 \AA$ or better from the PDB filtered at $90 \%$ sequence identity. From these models, several sets of non-overlapping
CVs were computed. In each set, CVs were generated from a different number of residues: three, nine, 15 and 21. All CV moduli were correlated to the standard DSSP annotation (Kabsch \& Sander, 1983). The analysis showed two distributions centred at two different means: $1.4 \AA$ for $\beta$-strands and $2.2 \AA$ for $\alpha$-helices. A Kruskal-Wallis equality of populations rank for comparing the medians of each data set revealed a significant difference (Kruskal \& Wallis, 1952). Indeed, large fragments tend to bend far away from the ideal description of an $\alpha$-helix or $\beta$-strand. Moreover, we could establish that the angle between consecutive vectors belonging to an $\alpha$-helix varies from 5 to $10^{\circ}$, whereas the range is from 50 to $55^{\circ}$ for consecutive vectors in a $\beta$-strand. Once again, the ranges are separate enough to avoid ambiguity.

The use of a single CV to capture the entire geometrical property of a secondary-structure element, as previously proposed (Sammito et al., 2013), was already sufficient to extract folds similar to a given template and generate libraries for fragment phasing. However, the new approach makes these vectors more comparable across different structures. As seen from our analysis, the CV distribution of tripeptides for each secondary-structure type presents small standard deviation, high kurtosis and low skewness. Curvature and bending inside a fragment are instead described by the moduli variations over the main chain observed in the discrete overlapping distribution.

The annotation algorithm in $A L E P H$ maps the distribution of overlapping CVs into a complete undirected edge-weighted graph, where a node represents a $\mathrm{CV}$ and an edge connecting two nodes stores the angle between the two connected CVs and their Euclidean distance. Each $\mathrm{CV}$ is assigned to an $\alpha$-helix (ah), $\beta$-strand (bs) or coil (coil) region by evaluating a

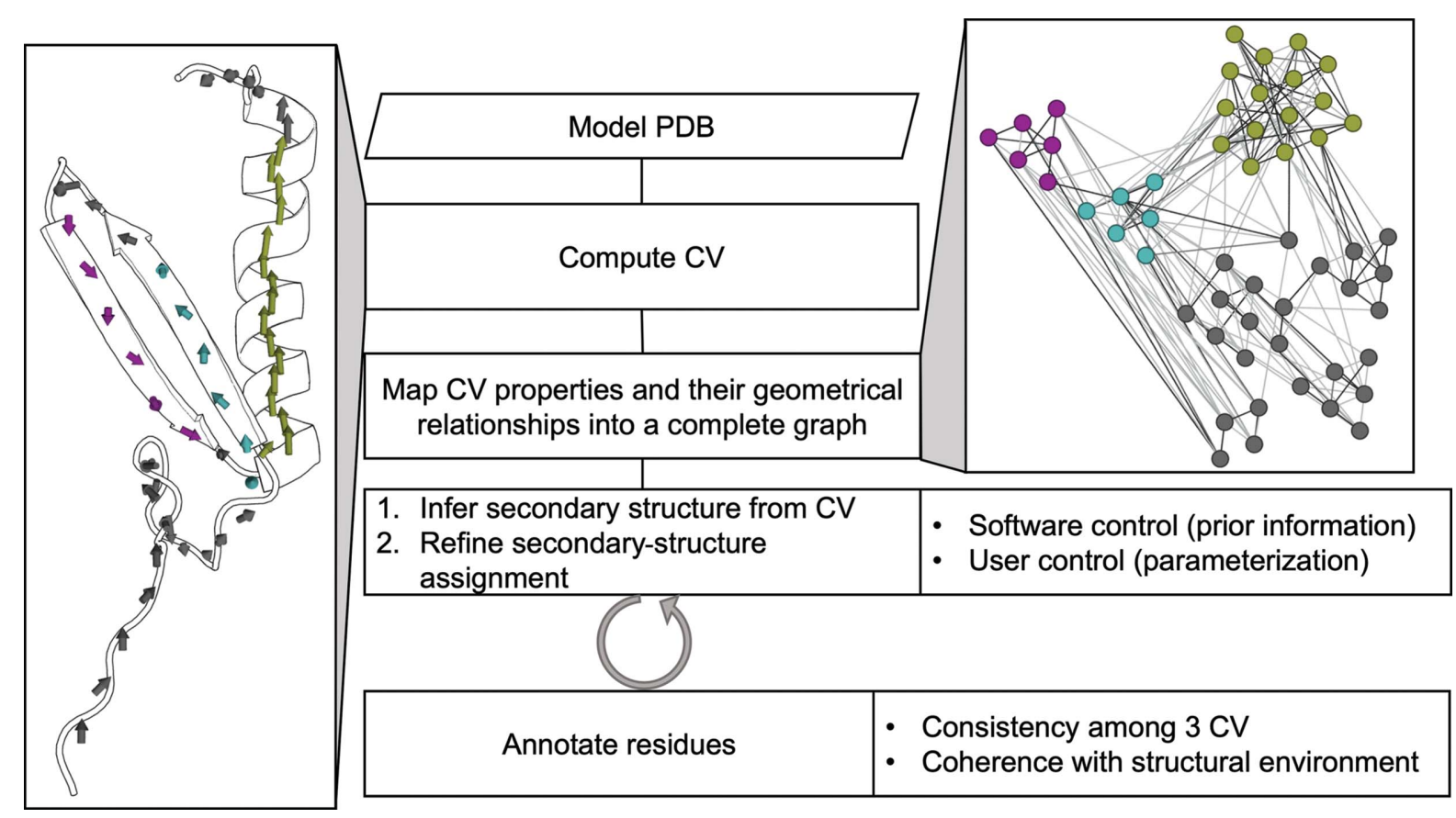

Figure 2

$A L E P H$ workflow for the annotation mode. 
penalty function, in which geometrical descriptors are used to determine the distance score of the $\mathrm{CV}$ from an ideal helix or strand. To evaluate the structural environment, the algorithm also includes distances and angles across different fragments. This pseudo-distance function has been heuristically estimated from the analysis of pre-annotated secondary-structure vectors. If the absolute difference between the two scores is larger than a chosen threshold that we call 'strictness', then the $\mathrm{CV}$ is annotated according to the lowest score. Otherwise, it will be annotated as a coil. The procedure involves several iterations in which the algorithm refines the weights and the values of each descriptor, improving the analysis of the structural environment.

The result at this point is an annotation for CVs, as each residue can participate in up to three different CVs. Translation into a residue annotation follows three rules.

(i) If all CVs in which a residue participates are annotated as either $a h$ or $b s$, so is the residue.

(ii) If two of the CVs in which a residue participates are annotated as $a h$ and none as $b s$, and the following residue is annotated as $a h$, then the current residue is annotated as $a h$.

(iii) A residue originally marked as coil will finally be annotated as $b s$ if it participates in two CVs annotated as $b s$ and none as $a h$, or if it participates in at least one CV annotated as $b s$ and one of the next or previous two residues is annotated as coil.

The last two rules are introduced to assign terminal residues in fragments separated by a short span of coil.

Once secondary-structure fragments have been annotated, their spatial relationship is annotated by mapping fragments onto a new graph where each fragment is represented by a supernode gathering all of its CV nodes. Edges relating these supernodes are annotated to describe their spatial relationships with the minimum, maximum and average of all angles and distances.

Edges are weighted by the inverse of the average distance and multiplied by a constant factor if the secondary structures connected are of the same type. In this way, fragments close in space will be related by higher weights and packing of $\beta$-sheets will be promoted. Edge weights prove useful for structure decomposition, as described in Section 3.2.1. Concomitantly, $\beta$-strands are packed into $\beta$-sheets and annotated in groups. Two strands will belong to the same sheet if at least $40 \%$ of the $\mathrm{CV}$ angles between the two fragments follow the empirical distribution observed for parallel and antiparallel $\beta$-strand CVs and their distance is lower than $6 \AA$.

Plots of the discrete distribution of $\mathrm{CV}$ moduli, plots of angles between consecutive $\mathrm{CV}$ s and plots of $\mathrm{C}^{\alpha}-\mathrm{C}^{\alpha}$ distances are generated, together with the corresponding tables reporting all numerical values. These plots can be used to identify anomalies, spot errors in the main chain and evaluate the goodness of the annotation, and are displayed in the graphical interface.

3.1.2. Examples: secondary-structure annotation for OppA and two helices of photosystem I. The structure of the peptide-binding protein OppA in complex with an endogenous peptide (PDB entry 1xoc) contains one chain of 520 amino acids and a peptide of nine amino acids (Levdikov et al., 2005). The space group is $P 2_{1} 2_{1} 2$ and the resolution is $1.55 \AA$. The $\alpha / \beta$ structure encompasses three domains according to the CATH server. DSSP annotates $29 \%$ helical residues and $23 \%$ $\beta$-sheet. ALEPH annotations of PDB entry 1xoc considering different strictness thresholds are shown in in Fig. 3. Selecting a lower strictness allows secondary-structure elements to be

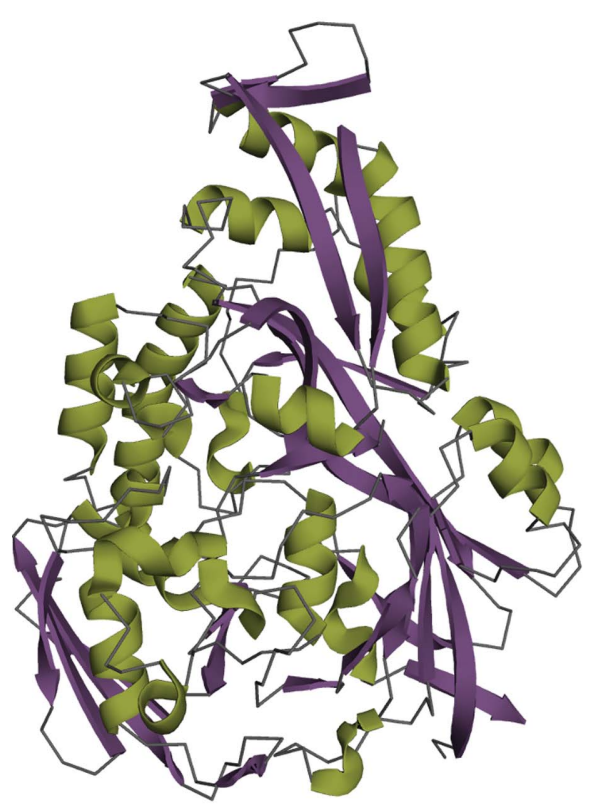

(a)

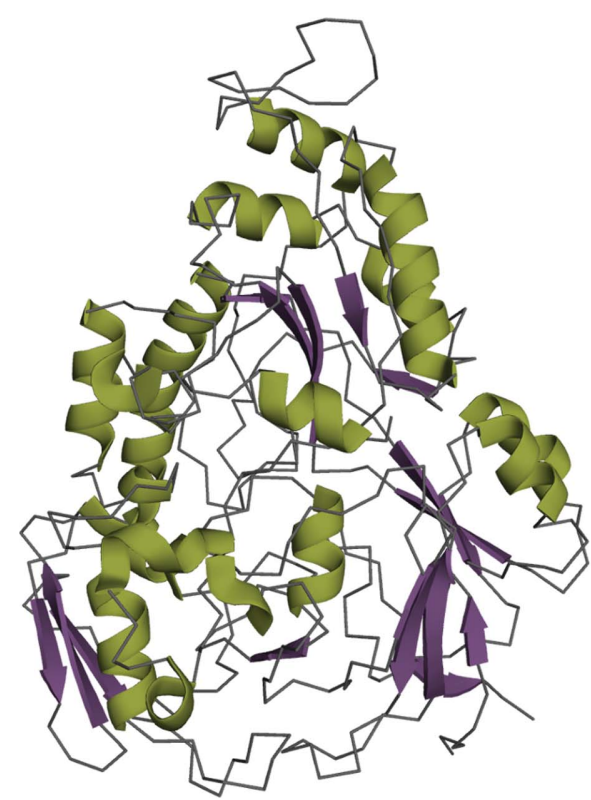

(b)

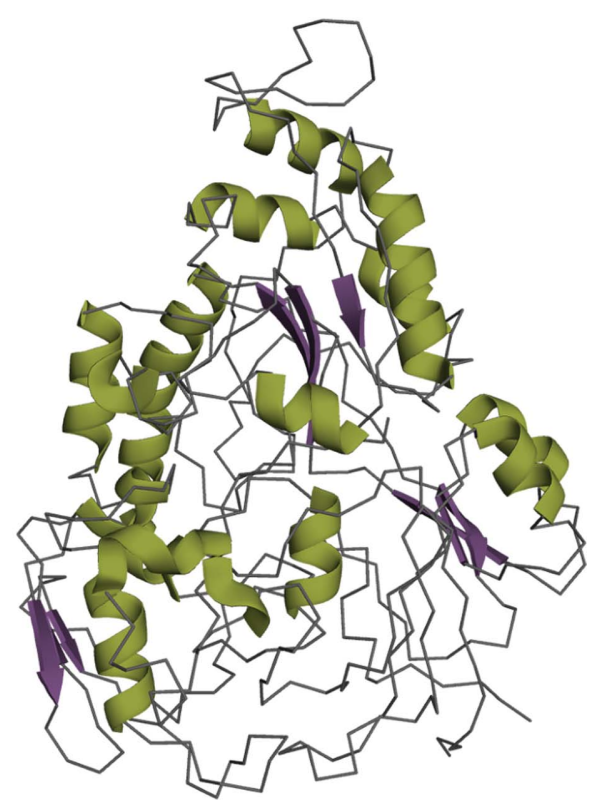

(c)

Figure 3

Comparison of the ALEPH annotation for PDB entry 1xoc with different parameterizations: strictness for $\alpha$-helices and $\beta$-strands set to $(a) 0.2$, $(b) 0.55$ and $(c)$ 0.6. The percentage of residues annotated as $\alpha$-helix and $\beta$-strand fragments are $(a) 33 \%$ and $33 \%,(b) 28 \%$ and $15 \%$ and $(c) 28 \%$ and $7 \%$, respectively. As the strictness threshold is increased, the algorithm annotates shorter, more ideal fragments. Colours represent secondary-structure types: green for $\alpha$-helices and purple for $\beta$-strands. 
extended, especially $\beta$-strands approximating bent fragments (Fig. 3c). In the case of PDB entry 1xoc a strictness of 0.55 (Fig. $3 b$ ) or higher will produce annotations that maintain the hydrogen-bond patterns establishing secondary-structure elements. At the lowest threshold of 0.2 (Fig. 3a) the hydrogen-bond pattern is occasionally broken; one residue is even found outside the secondary-structure area of the Ramachandran plot, while four residues are on the limit. More precise annotation can be found at higher strictnesses where the fragments tend to be shorter, accumulating less curvature. Depending on the intended application, one or other of the annotations might be preferred.

One example in which a less stringent description of the overall fold is preferable is the annotation of secondarystructure elements that present serious errors in the main chain. Although the fragment is an incorrect physical model, its approximation to a secondary-structure element can support the identification and correction of errors. Two fragments can be annotated as distorted helices (Fig. 4a): amino acids 201-227 and 298-317 from chain $A$ of the photosystem I supercomplex (PDB entry 2o01; Amunts et al., 2007). The direction of the carbonyl bonds is not parallel to the helical axis and this is reflected by shorter $\mathrm{CV}$ moduli and larger deviations in the angles between consecutive CVs than those found in $\alpha$-helices. The distances of consecutive $\mathrm{C}^{\alpha}$ atoms in the fragments are not constant.

The Ramachandran plot presents several outliers (Fig. 4b), which correspond to poor $\mathrm{CV}$ scores for $\alpha$-helices in the annotation. In the annotation procedure $A L E P H$ produces a file called strictnesses.pdb, which is displayed in the graphical interface. It shows the maximum strictness threshold required to annotate each residue as part of a secondarystructure element. Small values (red) in the difference between the scores for an $\alpha$-helix hypothesis and a $\beta$-strand hypothesis imply low confidence in the annotation; conversely, a larger value (blue) indicates a clear discrimination.

Hence, from analysis of the strictnesses.pdb output and inspection of the two helices of interest (Fig. 4c), we can observe large errors in both helices. Notice how CVs are sensitive to the misorientation of the carbonyl $\mathrm{O}$ atom and are less affected by a deformation of the helix turn.

Such poor geometry leads to differences in the annotations produced by DSSP and CaBLAM. Here, an analysis of the residue-based strictness output by $A L E P H$ could be a useful tool to spot the general secondary-structure features, distorted helical conformation and the local regions of low confidence, and hence the poor geometry.

\subsection{Decomposition through community clustering}

This section describes decomposition with $A L E P H$ of given protein folds into rigid subparts that will allow the comparison of proteins with overall similar folds but local dissimilarities. Network community clustering constitutes a set of algorithms that distribute all nodes in the graph into non-overlapping groups to maximize the modularity score (Newman, 2006b) of the graph. Formally, this score is defined as the fraction of the edges that fall within the given groups minus the expected fraction if the edges were distributed at random. Intuitively, it can be seen as a score that, if high, reflects dense connections

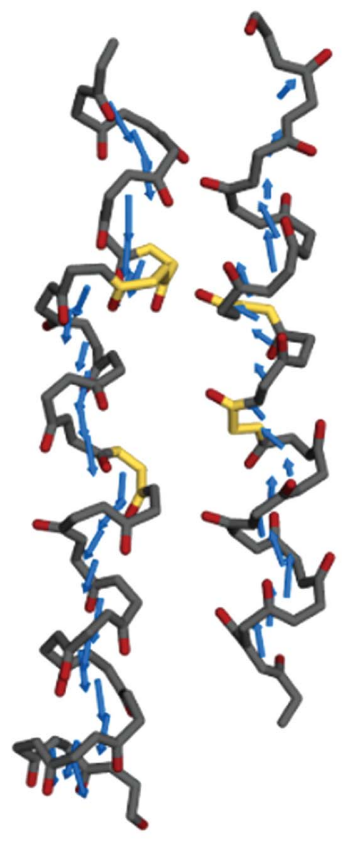

(a)

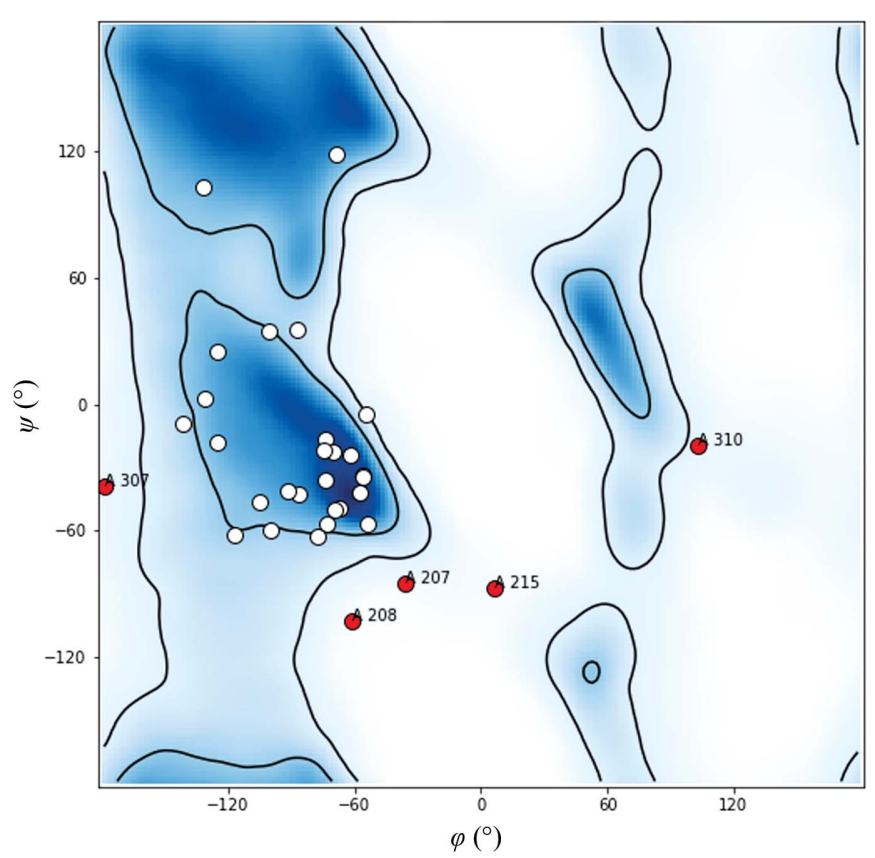

(b)

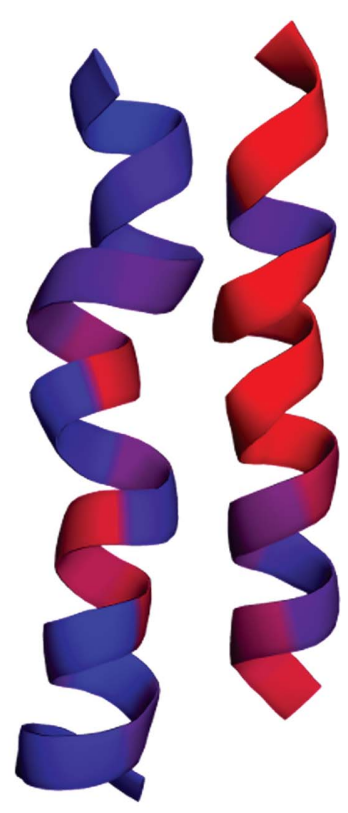

(c)

Figure 4

Annotation of severely distorted helices. (a) Main-chain backbone representation of the distorted helices from chain $A$ of photosystem I (PDB entry 2o01); carbonyl $\mathrm{O}$ atoms are coloured red. CVs are represented by blue arrows and the Ramachandran outliers are highlighted in yellow (amino acids 207, 208, 215, 307 and 310); they are displayed as red dots in the Ramachandran plot (b). (c) The strictness is annotated for each residue: blue indicates higher confidence than red. 
between the nodes within groups but sparse connections between nodes in different groups. In the context of MR, this decomposition can be used for the identification of compact rigid groups to refine their relative rotation and translation with respect to the other groups. It is known that protein domains or smaller motifs across homologous structures can move concertedly with respect to the overall fold; thus, even for pairs of structures sharing a very high sequence identity (above 60\%) it is common to observe deviations derived from conformational flexibility. Allowing the model additional degrees of freedom results in an increase in signal, enhancing the discrimination of the correct solution, improving the density map and providing a better partial solution for an eventual further search. The annotation of these groups with $A L E P H$ is used in the spherical mode of ARCIMBOLDO_ SHREDDER (Millán et al., 2018).

3.2.1. Implementation. Decomposition of a structure into compact folds is achieved by generating a graph in which each node represents a single secondary-structure element and the edges store statistical properties reflecting the geometrical relationship between the fragments. In particular, an average distance between two fragments is defined as the mean distance among all of the CVs involved in the pair. This number is used as the weight employed by the community clustering algorithm to optimize the group classification.
Although not directly corresponding to a physical property of the two fragments, it is a measure of proximity and allows the algorithms to generate compact folds.

The algorithm can force clustering to respect structural constraints, encouraging the formation of groups. For example, it is useful to cluster together the $\beta$-strands in a sheet. The decomposition algorithm optimizes the modularity score of the graph but can be biased to promote the formation of size-homogenous clusters containing the same number of secondary-structure elements, as discussed in Appendix $A$. These constraints are controlled by the edge weights in the graph. $A L E P H$ also provides a hierarchical decomposition in which the clustering procedure is iterated, increasing the number of groups to be output. This method generates a dendrogram in which each level corresponds to a progressive decomposition, ranging from all of the secondary-structure elements being included in one single cluster to each secondary-structure element belonging to a separate cluster. $A L E P H$ graphically represents the dendrogram and the hierarchical structural decomposition, opening a route to structural interpretations of the fold classification.

The workflow of the algorithm is shown in Fig. 5, illustrating the decomposition of the dimer formed by the wild-type diphtheria toxin (PDB entry 1fol) as discussed in the next section.
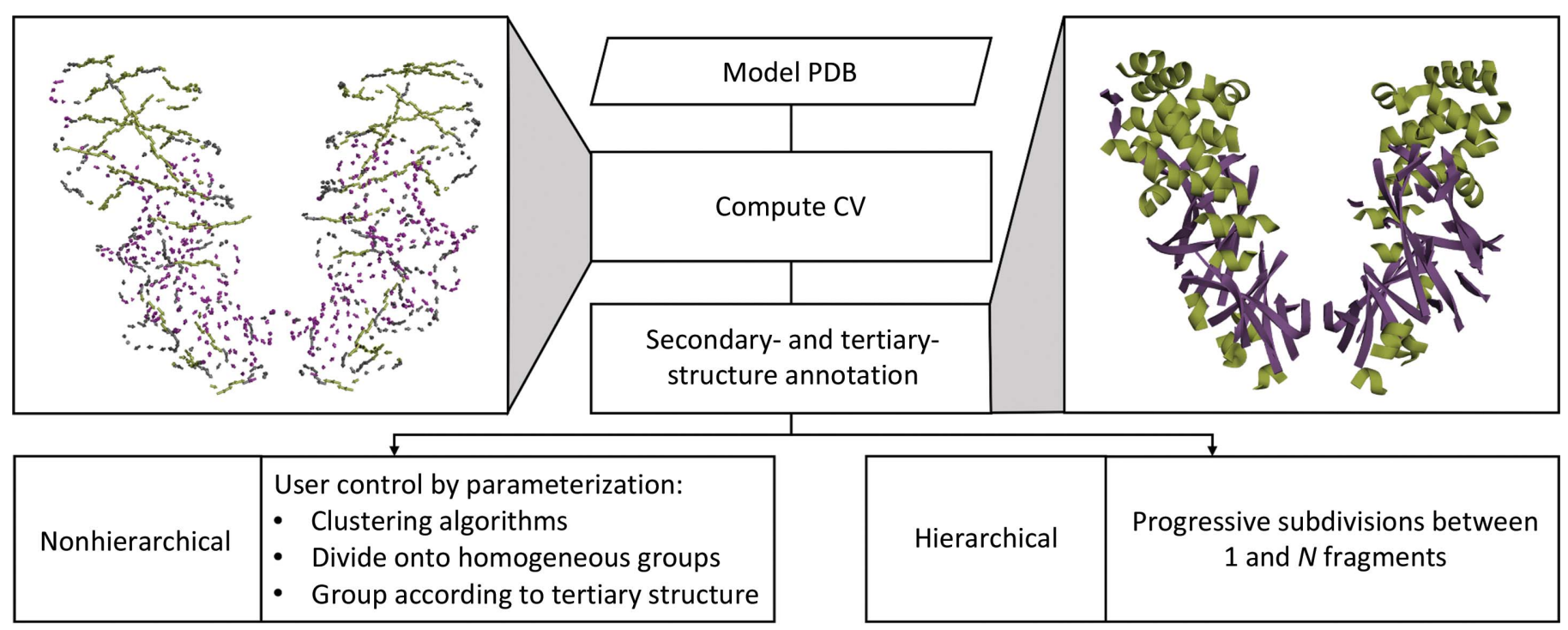

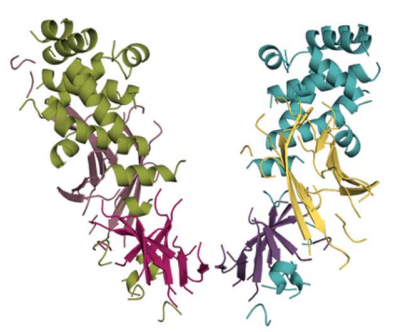

(b)

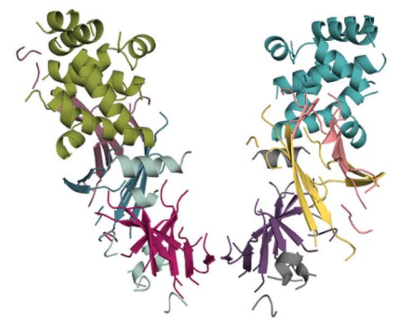

(c)
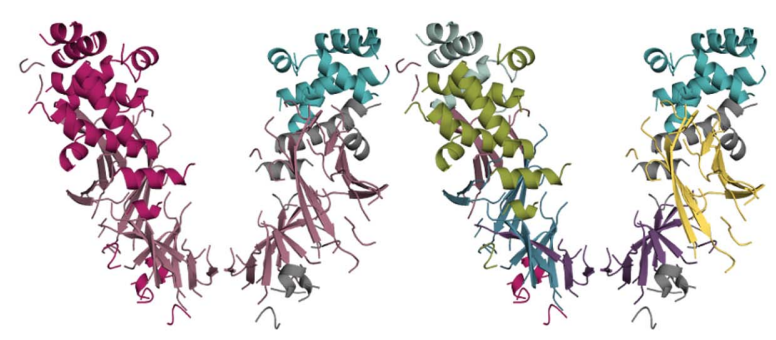

(d)

Figure 5

(a)

Workflow for the decomposition algorithm exemplified using the diphtheria toxin. (a) The program can alternatively perform a hierarchical or a nonhierarchical clustering. (b) Structural constraints are used, forming homogenous clusters and forcing $\beta$-strands to pack into $\beta$-sheets. ( $c$, $d$ ) Alternative decompositions performed $(c)$ with no constraints and $(d)$ hierarchically. 
3.2.2. Example: decomposition of the wild-type diphtheria toxin. The diphtheria toxin from corynephage beta (PDB entry 1f0l) is an ADP-ribosyltransferase which inhibits eukaryotic protein synthesis by inactivating elongation factor 2. The crystal structure, which was determined to $1.55 \AA$ resolution in space group $P 2_{1} 2{ }_{1} 2$, contains a homodimer. Each monomer is composed of 535 residues divided into three different domains, each belonging to a different superfamily: an N-terminal $\alpha-\beta$ complex, a central immunoglobulin-like domain and a C-terminal helical orthogonal bundle domain with globin-like topology.

Decompositions of the structure with different parameterizations were carried out to reveal the structural groups of the protein (Fig. 5). A nonhierarchical clustering, constraining groups to have a homogenous size and forcing strands to pack in $\beta$-sheets within the same cluster, resulted in a more biologically sensible classification, reflecting the three domains described above (Figs. $5 b$ and $5 c$ ). On the other hand, a hierarchical clustering in which the sub-decomposition was performed sequentially revealed different levels of compactness from the formation of the dimer to the nearest-neighbour fragment (Fig. 5d).

\subsection{Library generation}

In the context of fragment-based MR, the generation of a set of models representing the same small local fold may be used. Sequence-derived libraries from Rosetta are used in $a b$ initio models for phasing (Rigden et al., 2008). Such libraries provide sparse building blocks to approximately cover any part of a structure, whereas our libraries of superimposed models represent variations of a given geometry to find an accurate fragment. Previous knowledge can be used to filter the PDB and select the subset of structures from which to extract the library. The sequence-free extraction method is particularly useful for small and general folds that are ubiquitous in different protein families.

The generation of a library comprises five steps.

Step 1. Define the local folds to be extracted through a PDB template and select the parameterization.

Step 2. Parse and annotate the proteins stored as PDB files within a given directory or download a subset of structures based on a sequence or a family. Optionally, filter.

Step 3. Extract from the set of proteins every occurrence of the local fold, comparing and filtering with customizable thresholds.

Step 4. Superpose models to the original template and save to file, setting a common $B$ factor for all atoms.

Step 5. Cluster extracted models into geometrically similar groups.

It is possible to pre-annotate the whole PDB to speed up the procedure. Alternatively, the program annotates proteins during run time while executing a specific local fold search.

3.3.1. Implementation. A library generator has previously been introduced (Sammito et al., 2013), in which an entire secondary-structure fragment was mapped by a single $\mathrm{CV}$. The length of each $\mathrm{CV}$ was used to annotate the secondary- structure element and to perform extractions based on relative geometrical properties. This initial implementation was already able to grasp the general properties of fragments and local folds, allowing the extraction of libraries for the solution of unknown structures. The simplification of the geometrical properties to one $\mathrm{CV}$ per secondary-structure element did not allow the fine control that has now been achieved. In the current implementation (Fig. 6), the algorithm has evolved to enhance control through two types of vector relationships: angles and distances between vectors in the same fragment describe secondary structure, while those relating different fragments characterize the fold. The user can define different thresholds, expressed as percentages, for the two types of relationships. A higher threshold for secondary-structure vectors will restrict the extraction of models to contain geometrically closer fragments to those in the template input, for example avoiding the extraction of bent helices if the template provides straight helices. The tertiary-structure parameter controls the similarity in the arrangement of the fragments into a fold: the higher the threshold, the closer the relative distances and angles.

Once the template model (in PDB format) is annotated with CVs the fold is searched against the whole PDB (or any set of structures given in a folder). The user can limit this search, providing a CATH family (Dawson et al., 2017) or a $F A S T A$ sequence, which is used to perform a BLAST search against the PDB (https://www.rcsb.org/pages/webservices/ rest-search), sorting the results by $E$-value and retrieving the SCOP (Murzin et al., 1995) and CATH family from a candidate homologous structure with a minimum $E$-value of 0.005 . The list of unique SCOP and CATH identities is then used to filter the database during the search.

The structures in the database to be queried are annotated with CVs. The search is then performed in parallel, distributing computation over a grid network or a supercomputer facility, or just by multiprocessing on a single workstation. By default, if the target structure contains several equivalent monomers, only one will be evaluated. As folds can also be formed requiring the participation of two or more different chains (for example a coiled coil), this parameter can be changed if so wished.

The graph resulting from the template annotation is stored as a matrix, in which the cell at $(i, j)$ contains information about the angle and the distance between $\mathrm{CV}_{i}$ and $\mathrm{CV}_{j}$. Equivalent matrices are generated for every target structure in the database. The first diagonal would contain trivial selfrelations, but is instead used to store the CV length and secondary-structure annotation. The second diagonal stores the relationships between contiguous vectors. Therefore, identifying similar secondary-structure elements, regardless of their relative orientations and distances, will only require exploring the second diagonal in a linear time computation. The extraction of a template-like fold, considering the possible secondary-structure fragments identified, will require analysis of the corresponding off-diagonal cells. Even if the chosen fold is present in the target structure, the composite fragments can be rearranged in a different order or be separated by 


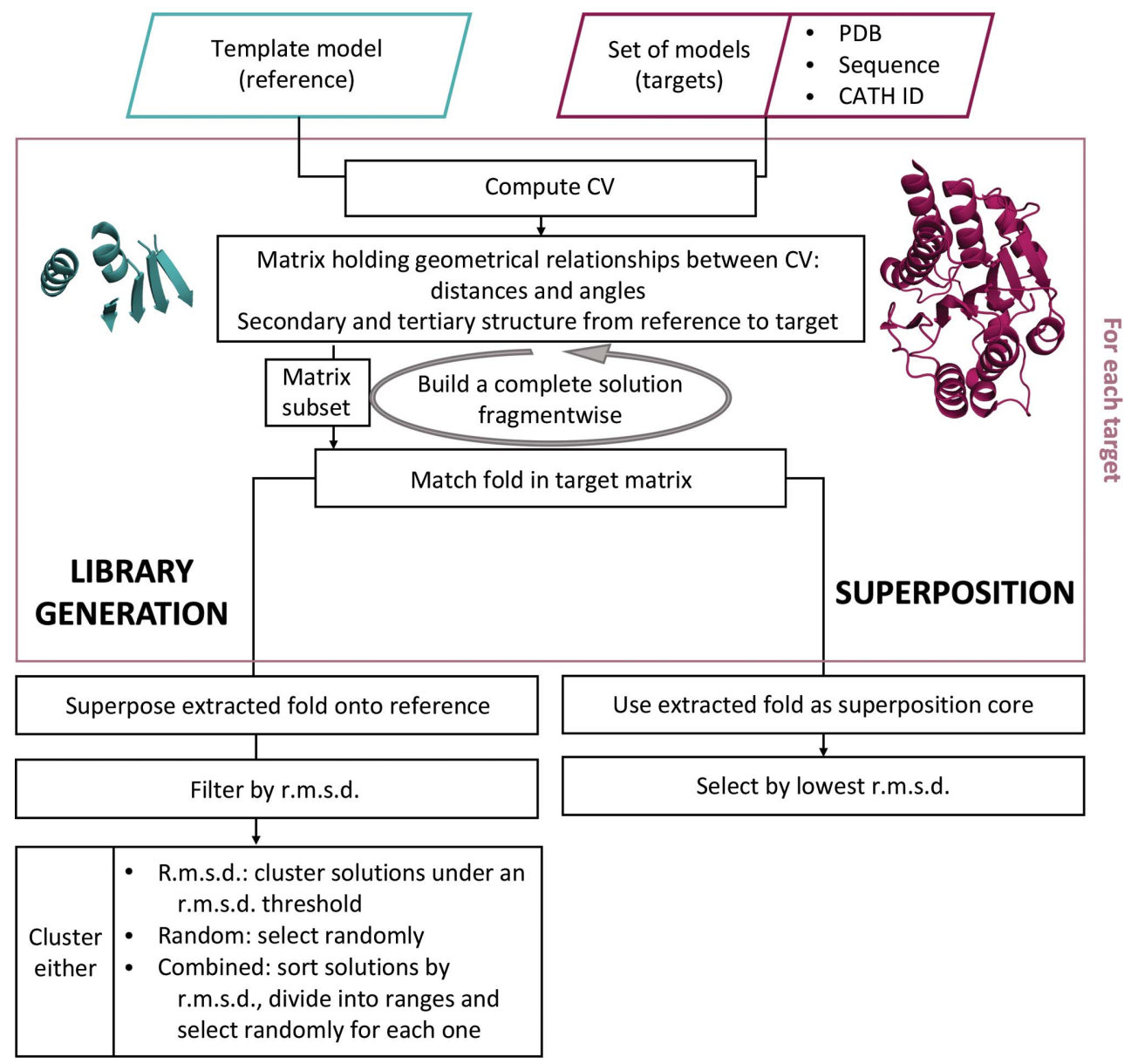

Figure 6

Library-extraction and superposition workflow. The minimal input required is a template in PDB format, which describes the fold to be extracted and the path to the stored PDB (or a subset database). For any other parameter a default is available, but the user might find it useful to adjust the strictness thresholds affecting the annotation of secondary-structure elements in both the template and the target and other parameters such as the intra-score and inter-score thresholds.

insertions. The problem of searching compatible fragments in the second diagonal is solved recursively, as shown in Fig. 7, and the resulting submatrices in the template and target structure are compared with a distance. The successful extraction of a given fold, if present in an annotated protein, is guaranteed by the completeness of the CV network (any pair of nodes is connected by an edge). ALEPH has to find any coherent combination of fragments that simultaneously satisfies the template matrix, in which not all relations should be equally weighted; for example, angle differences in coil regions might be less strict than among secondary-structure elements.

The user can configure structural conditions: a sequence matching the template size can be provided. The symbol $X$ indicates any valid residue. This parameter can be used to impose repeats or conserved cysteines. It is also possible to check for specific distances between $\mathrm{S}^{\gamma}$ atoms to enforce the presence of a disulfide bridge. Extracted models can be required to share the connectivity of the template, respecting the same $\mathrm{N}$-terminal to $\mathrm{C}$-terminal order in the fragments.
The extracted models are clustered into groups sharing closer geometry. This aims to reduce the number of models in the final library, avoiding redundant representation of the same variation of the fold. It also aims to better organize and discover fold properties, revealing the different types of observed conformation stored in the PDB. Alternative clustering algorithms are based on exhaustive pairwise comparison of r.m.s.d. between fragments, selection from an r.m.s.d. range to the template or a random selection of a subset of all possible occurrences. The choice should depend on the intended use of the library and the number of models to be extracted. While the first method does not involve a random selection, allowing reproducibility, and represents a finer criterion, it can have a long running time as the number of models extracted can be in the range of millions for very general ubiquitous folds. The other two methods are provided to perform faster clustering by sampling the space of the extracted models.

All of the models extracted and validated form a library that is superposed on the template and renamed according to the scheme pdbid_x_y yy $\cdot$ pdb, where pdbid is the original 


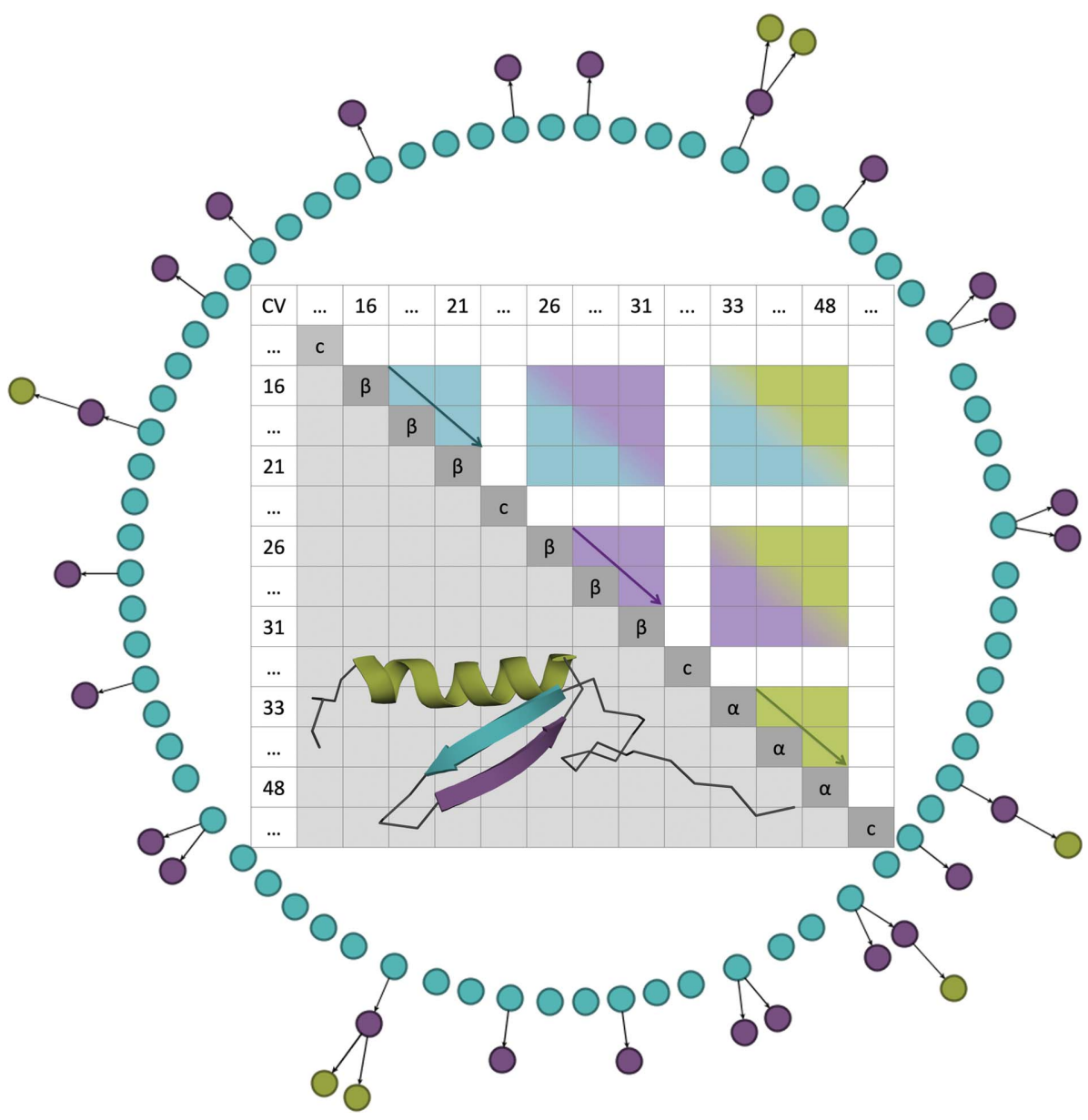

Figure 7

Scheme of the fold-extraction algorithm. The external graph represented in a circular layout is the forest of tree searches that are maintained in the memory by $A L E P H$ during extraction from a target structure (PDB entry 3to7). Each layer of the circle is coloured with the same colour as the template fragment (from PDB entry $4 \mathrm{e} 1 \mathrm{p}$ ) to extract. The light-blue $\beta$-strand is the first fragment to be found, followed by the second, purple $\beta$-strand and finally by the helix. Leaves in the external layer represent solutions satisfying all geometrical constraints according to the thresholds set $(60 \%$ for intra-vectors and inter-vectors). The half matrix displays the three fragments (compacting their lengths), showing triangle areas, coloured the same as the fragments, that carry the internal properties of each fragment and square areas where the geometrical relationships between two fragments are displayed using a colour gradient.

identifier of the PDB structure from which the model has been extracted, $x$ is the number of the structural model in the PDB entry (it differs from 0 only for NMR structures or ensemble models) and yyyy is an integer of a maximum of four digits that unequivocally identifies the model.

3.3.2. Example: phasing NovP from Streptomyces niveus with a library. To test the performance of the new librarygeneration algorithm in its use for phasing, we replicated our distributed libraries with fragments of the same secondary structure and generated new libraries of mixed $\alpha / \beta$ folds: ubiquitin-like and Rossmann folds. Here, we describe an example of an $\alpha / \beta$-fold library used to phase the $O$-methyltransferase NovP from $S$. niveus (PDB entry $2 \mathrm{wk} 1$; García et al., 2010). This protein is formed by a single monomer of 282 residues; the resolution of the data is $1.4 \AA$ and the space group is $P 2$.
The model used to create the library was extracted from the catechol $O$ methyltransferase from Rattus norvegicus (PDB entry 1vid; Vidgren et al., 1994), showing a typical Rossmann-fold domain consisting of a central core of parallel $\beta$-strands with antiparallel $\alpha$ helices on both sides. This fold is a very common fold found in many other protein families and thus is a good candidate for the generation of a general library of local protein folds. The particular fragment used as a template and shown in Fig. 8(a) contains four parallel $\beta$-strands and two $\alpha$-helices on one side (amino acids 44-57, 60-65, 71-79, 84-90, 111-116 and 136-140 from chain $A$ of PDB entry 1vid).

The data set used to generate the library is a subset of the PDB containing 18349 X-ray models filtered at 90\% sequence identity. We also removed structures deposited after the deposition of the test case (15 December 2009) to avoid bias in the results. We ran $A L E P H$, fixing strictness thresholds for $\alpha$-helices and $\beta$-strands of 0.5 and 0.3 , respectively. We set the secondarystructure score to $45 \%$ and the tertiarystructure score to $55 \%$, allowing higher local variation within each fragment while restricting the overall fold more. We also imposed a maximum limit of a $5.0 \AA$ r.m.s.d. to the template as a requirement to include models in the library. Clustering was not performed. The number of models that composed the superposed library output by $A L E P H$ was 9413. The minimum r.m.s.d. obtained against the template was $0.2 \AA$ for a model extracted from the template protein in a complex (PDB entry 3hvi). Library generation took approximately $9 \mathrm{~h}$ on a single workstation with eight cores. Some models were inspected and we could observe large rotations of the helices with respect to the $\beta$ sheet but preserving the distance from the plane defined by the helices to the $\beta$-sheet.

This library was used in ARCIMBOLDO_BORGES to phase the test protein PDB entry $2 \mathrm{wk} 1$. As the final refined structure of NovP was available, we could compute the wMPE of the output solutions and cluster phases in reciprocal space to count and identify the models from which the correct solution was found (Millán et al., 2020). ARCIMBOLDO_ $B O R G E S$, and hence the Phaser functions called, was run setting an initial r.m.s.d. of $0.6 \AA$. gyre refinement was skipped in the rotation step. After performing the translation search and packing check, the models were optimized with gimble 


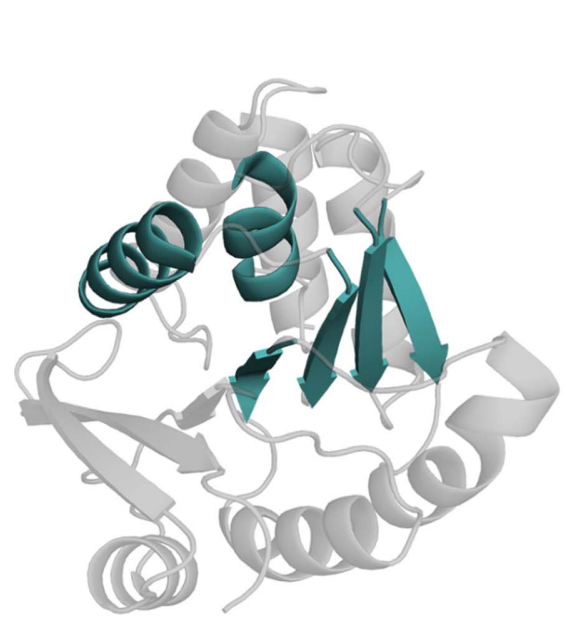

(a)

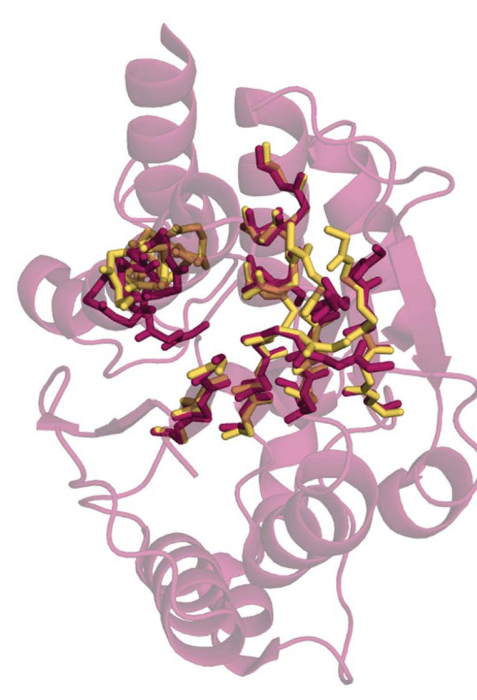

(b)

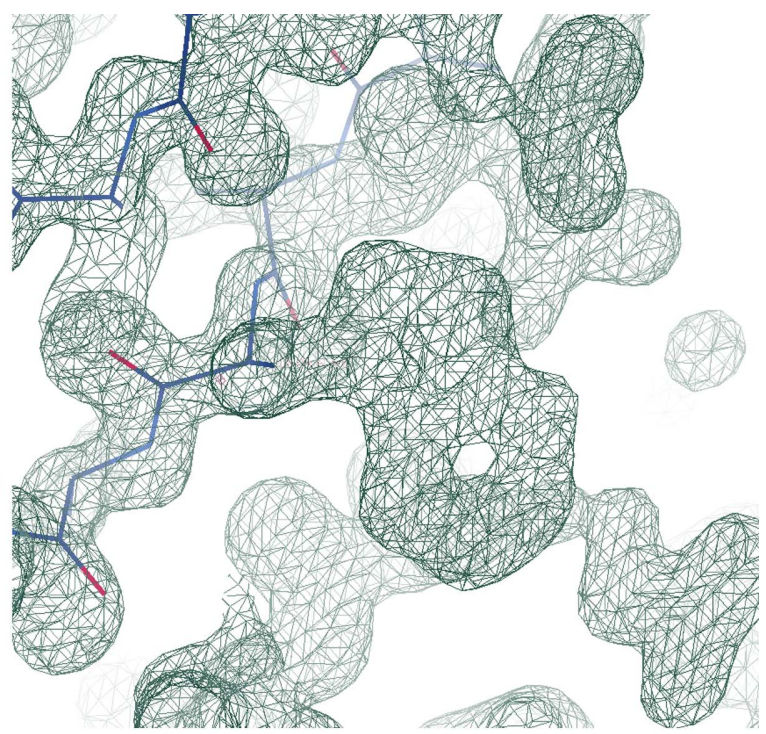

(c)

Figure 8

Solution of NovP with ARCIMBOLDO_BORGES. (a) Cartoon representation of the model, with PDB entry 1vid (grey) providing the template (turquoise) for library generation. (b) A model extracted from PDB entry $2 \mathrm{gdz}$ located by Phaser is shown in yellow and the final deposited structure of NovP (PDB entry 2wk1) is shown as a transparent red cartoon. (c) Detail of residue 119A: $F_{\mathrm{o}}$ FOM-weighted map at $3 \sigma$ showing clear electron density for the side chain of a tryptophan after performing density modification and autotracing with SHELXE.

refinement using the $A L E P H$ annotation, defining three independent rigid blocks: two formed by each helix and the third for the $\beta$-sheet. 25 nonrandom solutions were found with a wMPE against the deposited structure ranging from $71.6^{\circ}$ to $79.9^{\circ}$. All of them are related solutions, differing by less than $60^{\circ}$ from one other. The solutions are achieved with models extracted from 21 different deposited structures: PDB entries 2igt, 2pbf, 2wdq, 3e9n, 1yde, 2yxe, 3bzb, 1ej0, 1spx, 2gdz, 1hxh, 1cyd, 1y5m, 1db3, 2hrb, 2b4q, 2nm0, 1o5i, 1xu9, 3ip1 and $2 \mathrm{dm} 6$. The sequence identities of these structures to NovP are practically negligible. PDB entry $2 \mathrm{gdz}$, with a sequence identity of barely $5 \%$ and an overall r.m.s.d. of $7 \AA$ to NovP, rendered the fragment providing the best solution. The original Rossmann fold cut from PDB entry $2 \mathrm{gdz}$ shows an r.m.s.d. of $3.1 \AA$. After decomposition and rigid-group refinement with gimble in Phaser, all of the $\beta$-strands and one helix were placed correctly and only one helix was still partially misplaced.

To extend the placed fragment to a complete solution, SHELXE was set to iterate 15 cycles of density modification and autotracing. The solvent content was set to $44 \%$. The initial input model was trimmed to improve the correlation coefficient; in particular, SHELXE removed the misplaced helix. Data were extrapolated beyond the experimental resolution by up to $1 \AA$ (Usón et al., 2007). The new algorithm for tracing $\beta$-sheets in SHELXE was used to enhance tertiarystructure formation during tracing, as previously reported (Usón \& Sheldrick, 2018). ALEPH has generated libraries from which the parameters for the new SHELXE tracing algorithm have been deduced.

After six cycles of autotracing, ARCIMBOLDO_BORGES output a definite solution with a model extracted from PDB entry $2 \mathrm{gdz}$ (Figs. $8 b$ and $8 c$ ) that led to a SHELXE correlation coefficient of $34.9 \%$ with 199 residues traced. The completeness of the polypeptide trace was only $70 \%$, as by default ARCIMBOLDO stops after identifying a clear solution (CC > 30) where model building can be completed by another program, such as $A R P / w A R P$ (Chojnowski et al., 2020). In the electron-density map shown in Fig. $8(c)$, the side chains of aromatic amino acids are clearly visible. The initial r.m.s.d. of the extracted model belonging to the library to the final structure was $3.40 \AA$. After gimble refinement the model was improved, achieving a resolution of $2.18 \AA$, while the final r.m.s.d. after six cycles of tracing with $S H E L X E$ was $0.24 \AA$. Equivalent solutions were obtained from different structures presenting a lower initial r.m.s.d. to the target structure (for example a model from PDB entry 2pbf with an initial r.m.s.d. before gimble of $1.96 \AA$ ), but the ARCIMBOLDO_BORGES procedure stops as soon as a distinguishable solution is found and outputs the solution with the highest correlation coefficient.

\subsection{Superposition}

Structural superposition is one of the most frequent tasks that is routinely performed during the analysis and interpretation of macromolecular structures. Several algorithms are in use, from those based on least-squares optimization of the root-mean-square deviations among a common set of atoms as in $L S Q K A B$ (Kabsch, 1976) to maximum-likelihoodbased algorithms such as THESEUS (Theobald \& Wuttke, 2006). A very fast algorithm based on dynamic programming is currently distributed through $C C P 4$ under the name GESAMT (Krissinel, 2017), and molecular-modelling/ visualization programs have implemented their own algorithms such as the secondary-structure matching in Coot 


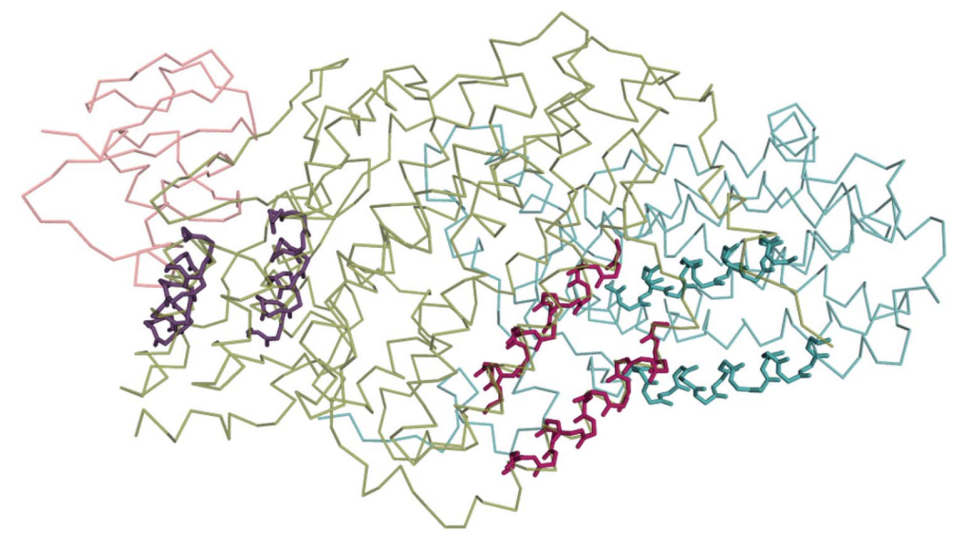

$(a)$

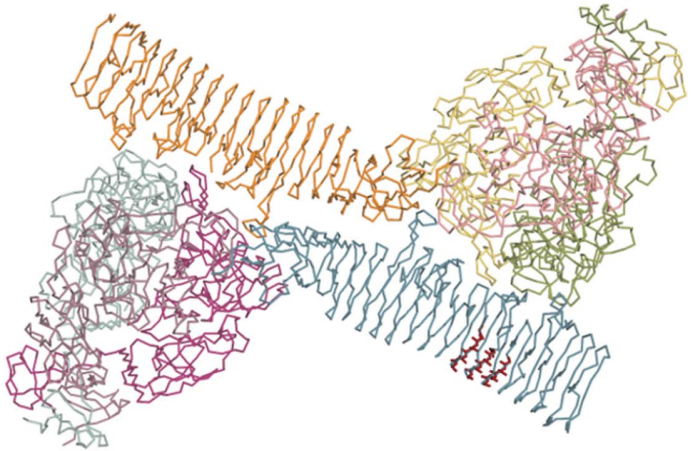

(d)

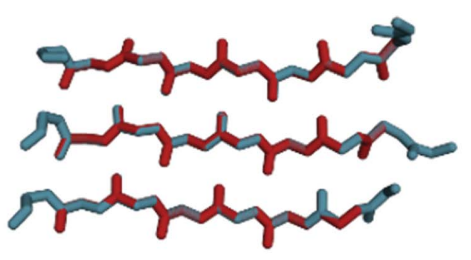

(e)

(b)

(c)

Figure 9

Example of the superposition of two small local folds onto complete structures. (a) Superposition by the GESAMT algorithm (purple) and the SSM algorithm (pink) of the library model and the protein with PDB entry $3 \mathrm{n} 1 \mathrm{z}$ coloured by chains: chain $A$, orange; chain $B$, blue; chain $C$, green. The fragment contained in the library was extracted from the area represented as blue sticks. (b) Close-up view of the GESAMT superposition. (c) View of the SSM superposition. (d) Superposition between a model from PDB entry 2iou represented as red sticks and the whole structure represented as ribbons (coloured by chain) as determined by the $A L E P H$ algorithm. (e) Stick representation of the superposed atoms.

(Emsley et al., 2010) and sequence-based and sequence-free matching in PyMOL (Schrödinger). TM-align (Zhang \& Skolnick, 2005) provides an alternative score encompassing the differences and extent of the match. ALEPH calculates this score for reference purposes, but its use in our context is limited.

Despite their high efficiency, these algorithms are optimized to superpose large connected domains or proteins and may sometimes run into difficulties when superposing small, disconnected folds or fragments onto a complete structure. For library generation in $A L E P H$ we have developed a simple procedure to address this very specific task, which is made available through the graphical application. The use of the library-generation procedure to perform superposition is displayed in the workflow in Fig. 6.

3.4.1. Implementation. Most of the algorithms available to superpose protein structures differ in the selection of a common core. ALEPH uses the library-extraction algorithm to find every possible correspondence of the local fold to a target. From this point, the core, rotation and the translation that minimize the r.m.s.d. are optimized. Part of this procedure is to iteratively weight corresponding pairs of atoms to the inverse of the variance of the atom around the average structure (Nilges et al., 1987) to improve the overall fit of the core. $A L E P H$ allows additional trimming at the extremities of each secondary-structure element in the core. In particular, for each fragment with more than five residues, a maximum number of three residues can be removed from the extremities. All combinations are tested and used to calculate an r.m.s.d. The lowest r.m.s.d. will determine the best core and superposition to be output. If the local fold matches the target structure at multiple non-overlapping sites, $A L E P H$ will output each of them separately. This feature may be useful to explore repetitions of a motif within a structure.

3.4.2. Examples: superposition of small helical folds and $\beta$-stranded folds onto structures. As an example, we show the superposition of small $\alpha$-helical and $\beta$-stranded fragments. A roto-translated model from a library of two parallel helices (Sammito et al., 2013), extracted from the monooxygenase hydroxylase with PDB code $3 \mathrm{n} 1 \mathrm{z}$, was superposed against the whole structure as in Fig. 9(a). The superposition should be able to relocate the fragment in its original position. Algorithms that are not designed for small fragments may fail, especially when their main chain is disconnected. In the first example, both the GESAMT (Fig. 9b) and SSM (Fig. 9c) algorithms, through SUPERPOSE (Krissinel \& Henrick, 2004), align fragments extracted from chain $B$ onto chain $A$ (with r.m.s.d.s of 2.02 and $0.75 \AA$, respectively). Chains $A$ and $B$ have different sizes and sequences and the identity between them is $17.9 \%$. Superposition with $A L E P H$ places the fragments exactly in their original location.

The second example tests a $\beta$-stranded local fold. Fig. $9(d)$ shows a ribbon representation of PDB entry 2iou. The complex is formed by three identical chains of major tropism 
Table 2

Summary of the libraries distributed with the current version of $A L E P H$.

The internal nomenclature $\mathrm{U}$ (up) and D (down) is used to describe the relative orientations of the fragments composing the fold; thus, UUU means three parallel fragments and UDU means antiparallel. BS, $\beta$-strand; AH, $\alpha$-helix.

\begin{tabular}{|c|c|c|c|c|c|c|}
\hline & Fold & $\begin{array}{l}\text { No. of template } \\
\text { residues }\end{array}$ & $\begin{array}{l}\text { Template } \\
\text { PDB code }\end{array}$ & $\begin{array}{l}\text { Template } \\
\text { residues }\end{array}$ & $\begin{array}{l}\text { No. of } \\
\text { models }\end{array}$ & $\begin{array}{l}\text { Novel structure } \\
\text { solved }\end{array}$ \\
\hline \multirow[t]{2}{*}{ Helices } & UD & 34 & $3 \mathrm{kfw}$ & $X 163-179, X 182-198$ & 6343 & 4gdo \\
\hline & UU & 32 & $3 \mathrm{rk} 2$ & $E 40-55, H 157-172$ & 11416 & $4 \mathrm{gn} 0$ \\
\hline & UUU & 20 & $1 \mathrm{c} 7 \mathrm{e}^{2}$ & $A 4-9, A 52-58, A 86-92$ & 5844 & \\
\hline & UUD & 20 & 4 aeq & $A 22-27, A 274-281, A 313-318$ & 7734 & \\
\hline & Sandwich BS UDU-UDU & 43 & $411 \mathrm{~h}$ & $A 18-24, A 32-38, A 61-67, A 70-76, A 83-90, A 99-105$ & 3069 & \\
\hline
\end{tabular}

determinant $P 1$ along with a single chain of the pertactin domain, consisting of a large $\beta$-helix fold of 536 amino acids. A model extracted from PDB entry 2iou (Fig. $9 e$ ) is contained in the three parallel $\beta$-strand library distributed through $C C P 4$ (Winn et al., 2011) with ARCIMBOLDO_BORGES (Sammito et al., 2015). In this case we could not superpose the 20 aminoacid fragments using other methods, while $A L E P H$ retrieved the correct superposition.

Finally, extraction of the library described in Sections 3.3.1 and 3.3.2 involves a superposition operation. According to HHpred (Zimmermann et al., 2018), the PDB contained 126 homologs to our template structure, PDB entry 1vid, all with a low sequence identity ranging from $5 \%$ to $18 \%$. ALEPH extracted library models from $61(48 \%)$ of them: those where the secondary-structure elements defined in the template were present.

Finding the optimal superposition of a fragment onto another structure is a task that can be performed using other fast and sophisticated methods such as GESAMT and SSM. The examples reported here illustrate the application of our program to the challenging case of small, discontinuous fragments. Depending on the use, one or the other of the algorithms should be advantageous.

\section{Distributed libraries}

Some of the libraries previously created with $A L E P H$ are distributed with $C C P 4$ for use as input search models in ARCIMBOLDO_BORGES. Recently, new libraries exploring more complex folds have been prepared and are available through our webpage. Table 2 lists the currently available libraries.

\section{Conclusion}

This work introduces the new software $A L E P H$, a graphbased tool to annotate secondary and tertiary structure from coordinates, decompose a structure into compact local small folds, extract local folds from a database of structures without using the sequence and generate libraries of such folds, which are especially useful as input search models for fragmentbased MR.

\section{APPENDIX $A$}

\section{A1. Cluster homogeneity in decomposition}

The decomposition algorithm can be biased to promote the formation of homogenous clusters containing the same number of secondary-structure elements. This is achieved by performing a hierarchical decomposition, and for each iteration $k$, corresponding to a clustering in $k$ groups, the modularity score of the decomposition graph is calculated as follows,

$$
Q_{k}=\frac{1}{2 m} \sum_{i j}\left(A_{i j}-\frac{k_{i} k_{j}}{2 m}\right)+\frac{\mu_{k}}{n}-\left[\frac{\sigma_{k}}{\mu_{k}(n-1)^{1 / 2}}\right],
$$

where $m$ is the number of edges in the decomposition graph, $\mathbf{A}$ is the adjacency matrix of the graph, the elements $A_{i j}$ of which are the total weight on edge $(i, j), k_{i}$ is the total weight adjacent to node $i$ and $k_{j}$ is computed similarly. $\mu_{k}$ is the mean number of secondary-structure elements among all of the decomposed clusters and $\sigma_{k}$ is the corresponding standard deviation. $N$ is the number of secondary-structure elements in the whole structure and $\sigma_{k} / \mu_{k}$ is the coefficient of variation. While this score promotes modularity, as previously defined (Newman, $2006 b$ ), it is biased toward larger clusters with a low dispersion of elements and thus more homogenously sized groups.

\section{A2. Distance score between matrices in extraction}

Some additional, more technical, details about the libraryextraction algorithm are given here. Firstly, the graph resulting from the template annotation is stored as a set of two symmetric matrices in which each cell at $(i, j)$ retains information about the angle and the distance between $\mathrm{CV}_{i}$ and $\mathrm{CV}_{j}$. The same pair of matrices are generated for every target structure in the database. $A L E P H$ also initializes a weight matrix $\mathbf{W}$ that enhances the contribution from smaller fragments, distinguishing them from the noise of random hits.

The main properties of the matrices are as follows.

(i) They are symmetric, as distance lengths and minimum angles within pairs of vectors are commutative operations.

(ii) The first diagonal stores the coordinates for any CV and its modulus, the annotated secondary-structure type and the information relative to any tertiary-structure fold with which it has been previously associated. 
(iii) The second diagonal stores the angles and the distances of each CV to the following CV. Secondary-structure elements remain defined by contiguous subsets of this diagonal. Two $\mathrm{CVs}$ are contiguous if the residues originating them are overlapping or contiguous.

(iv) Each secondary-structure element is uniquely referred to a set of matrix coordinates that enclose its $\mathrm{CVs}$ in the second diagonal.

(v) Identifying similar helices or strands, regardless of their relative orientations and distances, will only require exploring the second diagonal in a linear time computation.

(vi) Any cell that does not belong to the first diagonal stores the geometrical relationships between a specific pair of CVs; in particular, some of these cells store relationships among fragments.

A detailed description of the comparison between two matrices is now given describing the formulation of a distance score.

Suppose that $f_{1}, f_{2}, \ldots, f_{y}$ are fragments that have already been extracted and validated as one of the possible solution paths and $f_{x}$ is the new fragment that should be added to the current solution. Each fragment $f_{k}$ starts at the index s $f_{k}$ and ends at the index e $f_{k}$. Let $\mathbf{D}_{\mathrm{r}}$ and $\mathbf{T}_{\mathrm{r}}$ be the matrices $(n \times n)$ containing the distances and the angles of the CVs from the reference template, respectively, and $\mathbf{D}_{\mathrm{t}}$ and $\mathbf{T}_{\mathrm{t}}$ be the matrices for the distances and angles of the target structure from which fragments have been extracted. Then, let the new fragment $f_{x}$ define a sub-upper triangular matrix $\mathbf{D}_{\mathrm{r}}\left(\mathrm{s} f_{x}, \mathrm{e} f_{x}\right.$; $\left.\mathrm{s} f_{x}, \mathrm{e} f_{x}\right)$; this matrix contains all of the intra-geometrical distances for the CVs contained in the fragment $f_{i}$. Similarly, the sub-upper triangular matrix restricting $\mathbf{T}_{\mathrm{r}}$ can be defined. Both matrices have their diagonal coincident with a subset of the diagonal of their original matrices $\mathbf{D}_{\mathrm{r}}$ and $\mathbf{T}_{\mathrm{r}}$. The objective is to extract all of the possible submatrices of the same size in the matrices $\mathbf{D}_{\mathbf{t}}$ and $\mathbf{T}_{t}$, bounding their diagonals to be a subset of the $\mathbf{D}_{\mathrm{t}}$ and $\mathbf{T}_{\mathrm{t}}$ matrices.

The current extracted fragment $f_{x}$ also defines two new matrices $\mathbf{F}_{x k}$ and $\mathbf{G}_{x k}$ that contains only the distances and the angles, respectively, between CVs belonging to fragments $f_{x}$ and $f_{k}$, which is any of the previously found and validated fragments. Every time a submatrix is extracted it has to be compared with the corresponding submatrix from the template to establish whether its geometrical parameters are similar enough and can be included in the current solution path.

The program establishes the following algorithm to calculate the difference between two matrices.

(i) Let $\mathbf{A}$ and $\mathbf{B}$ be two matrices of the same size $(n \times n)$ both containing distances or angles.

(ii) $\mathbf{A}$ and $\mathbf{B}$ have to be normalized. If they contain distances,

$$
\mathbf{A}=\frac{A_{i j}}{\max \left(A_{i j}, B_{i j}\right)} \text { and } \mathbf{B}=\frac{B_{i j}}{\max \left(A_{i j}, B_{i j}\right)}
$$

If they contain angles,

$$
\mathbf{A}=\frac{A_{i j}}{\pi} \text { and } \mathbf{B}=\frac{B_{i j}}{\pi}
$$

(iii) A matrix difference $C=A_{i j}-B_{i j}$ is computed.

(iv) The weighted mean and the weighted standard deviation of $C$ are computed,

$$
\begin{gathered}
C_{\mu}=\frac{\sum C_{i j} W_{i j}}{\sum W_{i j}}, \\
C_{\sigma}=\frac{\sum\left(C_{i j}-C_{\mu}\right)^{2}-W_{i j}}{\sum W_{i j}} .
\end{gathered}
$$

(v) Distance is then defined as $d=1.0-\left[C_{\mu}+\left(\alpha C_{\sigma}\right)\right]+\varepsilon$, where $\alpha=1$ if $n \geq 20$, else $\alpha=2$, and $\varepsilon=0.1$ if a Mann-Whitney test (Mann \& Whitney, 1947) comparing $\mathbf{A}$ and $\mathbf{B}$ is true with a $p$-value of at least 0.1 , else $\varepsilon=-0.1$.

\section{Acknowledgements}

We thank George M. Sheldrick for helpful discussions.

\section{Funding information}

MDS acknowledges funding from the European Union's Horizon 2020 research and innovation programme under Marie Skłodowska-Curie grant agreement No. 790122. AM and $\mathrm{CM}$ wish to thank the Spanish Ministry of Economy and Competitiveness for grants BES-2017-080368 and BES-2015071397, respectively. RJB received a fellowship from FAPESP (16/24191-8 and 17/13485-3). This work was supported by grants BIO2015-64216-P, BIO2013-49604-EXP and MDM2014-0435-01 from the Spanish Ministry of Science and Innovation.

\section{References}

Amunts, A., Drory, O. \& Nelson, N. (2007). Nature, 447, 58-63.

Bastian, M., Heymann, S. \& Jacomy, M. (2009). Third International AAAI Conference on Weblogs and Social Media, pp. 361-362. Palo Alto: AAAI.

Bibby, J., Keegan, R. M., Mayans, O., Winn, M. D. \& Rigden, D. J. (2012). Acta Cryst. D68, 1622-1631.

Blondel, V. D., Guillaume, J.-L., Lambiotte, R. \& Lefebvre, E. (2008). J. Stat. Mech. 2008, P10008.

Caballero, I., Sammito, M., Millán, C., Lebedev, A., Soler, N. \& Usón, I. (2018). Acta Cryst. D74, 194-204.

Chojnowski, G., Choudhury, K., Heuser, P., Sobolev, E., Pereira, J., Oezugurel, U. \& Lamzin, V.S. (2020). Acta Cryst. D76, 248-260.

Clauset, A., Newman, M. E. J. \& Moore, C. (2004). Phys. Rev. E, 70, 066111.

Cock, P. J. A., Antao, T., Chang, J. T., Chapman, B. A., Cox, C. J., Dalke, A., Friedberg, I., Hamelryck, T., Kauff, F., Wilczynski, B. \& de Hoon, M. J. L. (2009). Bioinformatics, 25, 1422-1423.

Csardi, G. \& Nepusz, T. (2006). InterJ. Complex Syst. CX.18, 1695.

Dawson, N. L., Lewis, T. E., Das, S., Lees, J. G., Lee, D., Ashford, P., Orengo, C. A. \& Sillitoe, I. (2017). Nucleic Acids Res. 45, D289D295.

DiMaio, F., Terwilliger, T. C., Read, R. J., Wlodawer, A., Oberdorfer, G., Wagner, U., Valkov, E., Alon, A., Fass, D., Axelrod, H. L., Das, 
D., Vorobiev, S. M., Iwaï, H., Pokkuluri, P. R. \& Baker, D. (2011). Nature, 473, 540-543.

Emsley, P., Lohkamp, B., Scott, W. G. \& Cowtan, K. (2010). Acta Cryst. D66, 486-501.

Fujinaga, M. \& Read, R. J. (1987). J. Appl. Cryst. 20, 517-521.

Gómez García, I., Stevenson, C. E. M., Usón, I., Freel Meyers, C. L., Walsh, C. T. \& Lawson, D. M. (2010). J. Mol. Biol. 395, 390-407.

Headd, J. J., Echols, N., Afonine, P. V., Grosse-Kunstleve, R. W., Chen, V. B., Moriarty, N. W., Richardson, D. C., Richardson, J. S. \& Adams, P. D. (2012). Acta Cryst. D68, 381-390.

Holm, L. (2019). Bioinformatics, 35, 5326-5327.

Hunter, J. D. (2007). Comput. Sci. Eng. 9, 90-95.

Jenkins, H. T. (2018). Acta Cryst. D74, 205-214.

Kabsch, W. (1976). Acta Cryst. A32, 922-923.

Kabsch, W. \& Sander, C. (1983). Biopolymers, 22, 2577-2637.

Kalev, I., Mechelke, M., Kopec, K. O., Holder, T., Carstens, S. \& Habeck, M. (2012). Bioinformatics, 28, 2996-2997.

Karmali, A. M., Blundell, T. L. \& Furnham, N. (2009). Acta Cryst. D65, 121-127.

Krissinel, E. (2017). J. Comput. Sci. Appl. Inf. Technol. 2, 1-7.

Krissinel, E. \& Henrick, K. (2004). Acta Cryst. D60, 2256-2268.

Krissinel, E. \& Henrick, K. (2005). Computational Life Sciences, edited by M. R. Berthold, R. C. Glen, K. Diederichs, O. Kohlbacher \& I. Fischer, pp. 67-78. Berlin, Heidelberg: Springer.

Kruskal, W. H. \& Wallis, W. A. (1952). J. Am. Stat. Assoc. 47, 583-621. Levdikov, V. M., Blagova, E. V., Brannigan, J. A., Wright, L., Vagin, A. A. \& Wilkinson, A. J. (2005). J. Mol. Biol. 345, 879-892.

Liebschner, D., Afonine, P. V., Baker, M. L., Bunkóczi, G., Chen, V. B., Croll, T. I., Hintze, B., Hung, L.-W., Jain, S., McCoy, A. J., Moriarty, N. W., Oeffner, R. D., Poon, B. K., Prisant, M. G., Read, R. J., Richardson, J. S., Richardson, D. C., Sammito, M. D., Sobolev, O. V., Stockwell, D. H., Terwilliger, T. C., Urzhumtsev, A. G., Videau, L. L., Williams, C. J. \& Adams, P. D. (2019). Acta Cryst. D75, 861-877.

Lunin, V. Y. \& Woolfson, M. M. (1993). Acta Cryst. D49, 530-533.

Mann, H. B. \& Whitney, D. R. (1947). Ann. Math. Stat. 18, 50-60.

McCoy, A. J., Grosse-Kunstleve, R. W., Adams, P. D., Winn, M. D., Storoni, L. C. \& Read, R. J. (2007). J. Appl. Cryst. 40, 658-674.

McCoy, A. J., Oeffner, R. D., Millán, C., Sammito, M., Usón, I. \& Read, R. J. (2018). Acta Cryst. D74, 279-289.

McCoy, A. J., Oeffner, R. D., Wrobel, A. G., Ojala, J. R. M., Tryggvason, K., Lohkamp, B. \& Read, R. J. (2017). Proc. Natl Acad. Sci. USA, 114, 3637-3641.

Millán, C., Jiménez, E., Schuster, A., Diederichs, K. \& Usón, I. (2020). Acta Cryst. D76, 209-220.

Millán, C., Sammito, M. \& Usón, I. (2015). IUCrJ, 2, 95-105.

Millán, C., Sammito, M. D., McCoy, A. J., Nascimento, A. F. Z., Petrillo, G., Oeffner, R. D., Domínguez-Gil, T., Hermoso, J. A., Read, R. J. \& Usón, I. (2018). Acta Cryst. D74, 290-304.

Murzin, A. G., Brenner, S. E., Hubbard, T. \& Chothia, C. (1995). J. Mol. Biol. 247, 536-540.

Newman, M. E. J. (2006a). Phys. Rev. E, 74, 036104.

Newman, M. E. J. (2006b). Proc. Natl Acad. Sci. USA, 103, 8577-8582.

Newman, M. E. J. \& Girvan, M. (2004). Phys. Rev. E, 69, 026113.

Nilges, M., Clore, G. M. \& Gronenborn, A. M. (1987). FEBS Lett. 219, $11-16$.

Oeffner, R. D., Afonine, P. V., Millán, C., Sammito, M., Usón, I., Read, R. J. \& McCoy, A. J. (2018). Acta Cryst. D74, 245-255.

Oeffner, R. D., Bunkóczi, G., McCoy, A. J. \& Read, R. J. (2013). Acta Cryst. D69, 2209-2215.

Pauling, L. \& Corey, R. B. (1951). Proc. Natl Acad. Sci. USA, 37, 251256.

Pauling, L., Corey, R. B. \& Branson, H. R. (1951). Proc. Natl Acad. Sci. USA, 37, 205-211.

Pedregosa, F., Varoquaux, G., Gramfort, A., Michel, V., Thirion, B., Grisel, O., Blondel, M., Müller, A., Nothman, J., Louppe, G.,
Prettenhofer, P., Weiss, R., Dubourg, V., Vanderplas, J., Passos, A., Cournapeau, D., Brucher, M., Perrot, M. \& Duchesnay, É. (2012). arXiv:1201.0490.

Pereira, J. \& Lamzin, V. S. (2017). IUCrJ, 4, 657-670.

Pons, P. \& Latapy, M. (2005). Computer and Information Sciences ISCIS 2005, edited by P. Yolum, T. Güngör, F. Gürgen \& C. Özturan, pp. 284-293. Berlin, Heidelberg: Springer.

Pröpper, K., Meindl, K., Sammito, M., Dittrich, B., Sheldrick, G. M., Pohl, E. \& Usón, I. (2014). Acta Cryst. D70, 1743-1757.

Raghavan, U. N., Albert, R. \& Kumara, S. (2007). Phys. Rev. E, 76, 036106.

Ramachandran, G. N., Ramakrishnan, C. \& Sasisekharan, V. (1963). J. Mol. Biol. 7, 95-99.

Read, R. J. \& McCoy, A. J. (2016). Acta Cryst. D72, 375-387.

Reichardt, J. \& Bornholdt, S. (2006). Phys. Rev. E, 74, 016110.

Richardson, J. S., Williams, C. J., Hintze, B. J., Chen, V. B., Prisant, M. G., Videau, L. L. \& Richardson, D. C. (2018). Acta Cryst. D74, 132-142.

Rigden, D. J., Keegan, R. M. \& Winn, M. D. (2008). Acta Cryst. D64, 1288-1291.

Rodríguez, D., Sammito, M., Meindl, K., de Ilarduya, I. M., Potratz, M., Sheldrick, G. M. \& Usón, I. (2012). Acta Cryst. D68, 336343.

Rodríguez, D. D., Grosse, C., Himmel, S., González, C., de Ilarduya, I. M., Becker, S., Sheldrick, G. M. \& Usón, I. (2009). Nat. Methods, 6, 651-653.

Rosvall, M. \& Bergstrom, C. T. (2008). Proc. Natl Acad. Sci. USA, 105, 1118-1123.

Roy, A., Kucukural, A. \& Zhang, Y. (2010). Nat. Protoc. 5, 725-738.

Sammito, M., Meindl, K., de Ilarduya, I. M., Millán, C., ArtolaRecolons, C., Hermoso, J. A. \& Usón, I. (2014). FEBS J. 281, 4029 4045.

Sammito, M., Millán, C., Frieske, D., Rodríguez-Freire, E., Borges, R. J. \& Usón, I. (2015). Acta Cryst. D71, 1921-1930.

Sammito, M., Millán, C., Rodríguez, D. D., de Ilarduya, I. M., Meindl, K., De Marino, I., Petrillo, G., Buey, R. M., de Pereda, J. M., Zeth, K., Sheldrick, G. M. \& Usón, I. (2013). Nat. Methods, 10, 10991101.

Sheldrick, G. M. (2002). Z. Kristallogr. 217, 644-650.

Sheldrick, G. M. (2010). Acta Cryst. D66, 479-485.

Shrestha, R. \& Zhang, K. Y. J. (2015). Acta Cryst. D71, 304-312.

Tannenbaum, T., Wright, D., Miller, K. \& Livny, M. (2001). Beowulf Cluster Computing with Linux, edited by T. Sterling, pp. 307-350. Cambridge: MIT Press.

Theobald, D. L. \& Wuttke, D. S. (2006). Bioinformatics, 22, 2171 2172.

Touw, W. G., Baakman, C., Black, J., te Beek, T. A. H., Krieger, E., Joosten, R. P. \& Vriend, G. (2015). Nucleic Acids Res. 43, D364D368.

Usón, I. \& Sheldrick, G. M. (2018). Acta Cryst. D74, 106-116.

Usón, I., Stevenson, C. E. M., Lawson, D. M. \& Sheldrick, G. M. (2007). Acta Cryst. D63, 1069-1074.

Vidgren, J., Svensson, L. A. \& Liljas, A. (1994). Nature, 368, 354 358.

Walt, S. van der, Colbert, S. C. \& Varoquaux, G. (2011). Comput. Sci. Eng. 13, 22-30.

Winn, M. D., Ballard, C. C., Cowtan, K. D., Dodson, E. J., Emsley, P., Evans, P. R., Keegan, R. M., Krissinel, E. B., Leslie, A. G. W., McCoy, A., McNicholas, S. J., Murshudov, G. N., Pannu, N. S., Potterton, E. A., Powell, H. R., Read, R. J., Vagin, A. \& Wilson, K. S. (2011). Acta Cryst. D67, 235-242.

Zhang, Y. \& Skolnick, J. (2005). Nucleic Acids Res. 33, 2302-2309.

Zhou, J. \& Grigoryan, G. (2015). Protein Sci. 24, 508-524.

Zimmermann, L., Stephens, A., Nam, S.-Z., Rau, D., Kübler, J., Lozajic, M., Gabler, F., Söding, J., Lupas, A. N. \& Alva, V. (2018). J. Mol. Biol. 430, 2237-2243. 